\title{
«Somos estudiantes del campo»: identificaciones de jóvenes rurales en Entre Ríos (Argentina)
}

\author{
María-Emilia Schmuck, Ph. D. ${ }^{a}$ \\ Instituto de Estudios Sociales, Argentina
}

emilia.schmuck@gmail.com

\section{Resumen (analítico)}

La extensión de la escolaridad en Argentina y la creación de escuelas secundarias en el campo ha significado transformaciones en el espacio rural y las juventudes rurales. A partir de una investigación realizada desde el enfoque histórico-etnográfico en el norte de la provincia de Entre Ríos, advertimos que la escuela secundaria es el espacio privilegiado de sociabilidad de las y los jóvenes y nos centramos en la Fiesta del Estudiante Rural para comprender los procesos de identificación de estas juventudes. La condición de juventud y la condición de estudiante están estrechamente implicadas en el espacio rural y las y los jóvenes construyen identificaciones por oposición a sus pares urbanos y en relación con el campo, entendido en tanto paisaje natural escindido de las actividades productivas y laborales de la ruralidad.

\section{Palabras clave}

Joven rural; estudiante de secundaria; educación rural; Argentina.

\section{Thesauro}

Tesauro de Ciencias Sociales de la Unesco.

\section{Para citar este artículo}

Schmuck, M. E. (2022). «Somos estudiantes del campo»: identificaciones de jóvenes rurales en Entre Ríos (Argentina). Revista Latinoamericana de Ciencias Sociales, Niñez y Juventud, 20(1), 1-26. https://dx.doi.org/10.11600/rlcsnj.20.1.4865

\section{Historial}

Recibido: 01.06.2021

Aceptado: 08.10.2021

Publicado: 09.12.2021

\section{Información artículo}

Este artículo presenta hallazgos de la tesis doctoral "Somos jóvenes y estudiantes del campo»: una etnografía sobre experiencias formativas y educación secundaria en el norte entrerriano (Universidad Nacional de Entre Ríos), que fue aprobada en mayo de 2020 y cuyo trabajo de campo se realizó entre febrero de 2017 y diciembre de 2019. La investigación fue financiada a partir de una beca doctoral otorgada por Conicet y desarrollada en el Instituto de Estudios Sociales, dependiente de Conicet y la Universidad Nacional de Entre Ríos, en Argentina. Área: antropología. Subárea: antropología de la educación. 


\section{«We are students of the countryside». Identities and identifications of rural youth in Entre Ríos (Argentina)}

\section{Abstract (analytical)}

The rollout of public education across the country in Argentina and the building of secondary schools in the countryside generated transformations for rural areas and rural youth. This study uses a historical-ethnographic approach to conduct research in the northern area of the province of Entre Ríos. The authors identified that secondary school is a privileged space for the social development of young people, focusing on the Rural Student Festival to understand these youth identities. The youth condition and the student condition are closely linked to rural areas. Rural young people construct identifications that are in opposition to urban students and aligned with the countryside, which they understand as a natural landscape that is unrelated to agricultural and labor activities.

Keywords

Rural youth; secondary school students; rural education; Argentina.

\section{«Somos estudantes de campo». Identificações de jovens rurais em Entre Ríos (Argentina)}

\section{Resumo (analítico)}

A extensão da escolaridade na Argentina e a criação de escolas secundárias no campo geraram transformações no meio rural e na juventude rural. A partir de uma investigação realizada a partir de uma abordagem histórico-etnográfica no norte da província de Entre Ríos, afirmamos que o ensino médio é o espaço privilegiado de sociabilidade dos jovens e nos concentramos no Festival do Estudante Rural para compreender as identidades destes jovens. A condição de jovem e a condição de estudante estão intimamente envolvidas no meio rural e os jovens constroem identificações em oposição aos estudantes urbanos e em relação ao campo, entendido como paisagem natural alheia às atividades produtivas e laborais do campo.

\section{Palavras-chave}

Juventude rural; estudante do ensino médio; educação rural; Argentina.

\section{Información autora}

[a] Becaria postdoctoral en el Instituto de Estudios Sociales del Consejo Nacional de Investigaciones Científicas y Técnicas de Argentina (Conicet) y la Universidad Nacional de Entre Ríos. Licenciada en Comunicación Social de la Universidad Nacional de Entre Ríos. Doctora en Ciencias Sociales de la Universidad Nacional de Entre Ríos. iD 0000-0003-2982-6394. H5: 1. Correo electrónico: emilia.schmuck@gmail.com 


\section{Introducción}

$\mathbf{E}_{\text {ción de las juventudes rurales. Para ello, retomamos parte de los resultados de una }}$ investigación ${ }^{1}$ realizada desde el enfoque histórico-etnográfico en la que analizamos la configuración de las experiencias formativas de las juventudes en un contexto de extensión de la obligatoriedad escolar y creación de escuelas secundarias en los espacios rurales en Argentina.

A lo largo de la historia de los estudios sociales en América Latina se ha tendido a invisibilizar a las y los sujetos juveniles en el campo (González-Cangas, 2003; Kessler, 2007), lo que se relaciona en parte con las directrices dominantes en los estudios provenientes de las ciencias sociales rurales que tendieron a considerar la inevitable urbanización total y la consiguiente migración de los jóvenes a las ciudades. En Argentina, podemos señalar que las y los jóvenes que viven, estudian o trabajan en contextos rurales no emergen con frecuencia en la escena pública nacional ni ocupan un lugar protagónico en los estudios sociales (Schmuck, 2019a), aunque en los últimos años la ruralidad se destaca como una de las emergencias temáticas en los estudios y reuniones académicas sobre juventudes (Alvarado et al., 2021). Entre las investigaciones pioneras en el país encontramos trabajos que se proponen superar el estado de vacancia y buscan describir la situación de las y los jóvenes en todo el territorio (Caputo, 2001; Román, 2003; Román, 2011), otros que profundizan en determinadas regiones (Bruniard et al., 2007; Calvo et al., 2015) y, principalmente en la última década, valiosas investigaciones que parten de entender que la juventud y la ruralidad son categorías polisémicas y estudian jóvenes en una pluralidad de contextos étnico raciales, culturales, de clase y territorios (Barés et al., 2020).

\footnotetext{
${ }^{1}$ La investigación se enmarcó en la realización de la tesis doctoral titulada «Somos jóvenes y estudiantes del campo»: una etnografía sobre experiencias formativas y educación secundaria en el norte entrerriano (Schmuck, 2020), que fue financiada a partir de una beca de Conicet en el Instituto de Estudios Sociales de la Universidad Nacional de Entre Ríos. En el trabajo nos preguntarnos por los procesos de identificación de las juventudes rurales, dimensión que en este artículo nos proponemos desarrollar con detenimiento, al tiempo que abordamos las transformaciones y continuidades en las experiencias formativas de las jóvenes generaciones en relación con las generaciones que las anteceden y atendiendo a las desigualdades de género.
} 
Entre estos últimos estudios, aquí interesa recuperar, en primer lugar, una investigación sobre jóvenes de familias que se dedican a la tarefa (cosecha de yerba mate) en barrios periurbanos de la provincia de Misiones; esta considera el lugar protagónico que tiene la emoción del sufrimiento en la conformación de sus identidades y observa diversas estrategias ligadas a la resistencia por asumir la identidad tarefera sufrida por sus padres y madres (Roa, 2013). Por otra parte, una investigación sobre juventudes de familias campesinas rurales de Chubut y Río Negro problematiza qué es ser joven en estos territorios y señala que el establecimiento de las escuelas secundarias en la zona - que además profundizó el acceso, el uso y consumo de las nuevas tecnologías - contribuyó a crear lo juvenil (Barés, 2016), siendo la escuela un espacio de socialización donde las y los jóvenes tienen sus rutinas diferenciadas y consumos específicos. En un trabajo que establece cruces entre estas dos últimas investigaciones, las autoras señalan que las identidades juveniles, que se constituyen en relación con diversos trayectos rural-urbanos, se vinculan con estigmatizaciones simbólicas propias de sus pertenencias étnico-raciales-territoriales. Sin embargo, advierten que es posible divisar modos creativos en que habitan esta estigmatización en el territorio, el trabajo, la escuela o el barrio, al tiempo que destacan que las y los jóvenes dicen «hallarse en el campo», lo que se entiende como un «sentirse cómodo/a» en el territorio como fundamento existencial que encarna las identidades juveniles rurales (Barés \& Roa, 2020).

En el mismo sentido se expresa un trabajo reciente pero desarrollado en Colombia, particularmente en la región de Cundinamarca, que destaca la centralidad de las instituciones escolares urbanas a la que asisten jóvenes de origen rural en la reconfiguración de sus identidades. En este estudio se señala que, si acaso las limitaciones al acceso a expresiones culturales, artísticas y deportivas los ponen en desventaja frente a jóvenes urbanos y entonces vivencian sensaciones humillación, también agencian oportunidades, destacan las bondades de vivir en el campo y reafirman su arraigo al territorio y las actividades productivas, aunque al finalizar su trayectoria escolar decidan emigrar a la ciudad (CifuentesGarzón, 2021).

Particularmente en relación con nuestra perspectiva de trabajo, también interesa destacar los aportes de investigaciones sobre juventudes rurales y educación realizadas en Argentina desde el enfoque etnográfico y los estudios socioantropológicos (Ambrogi, 2020; Cragnolino, 2017; Gili-Diez, 2019; Gómez \& Porta, 2018; Hirsch, 2020; Padawer et al., 2015; Romero-Acuña, 2018). Entre estas, aquí nos interesa particularmente desarrollar una indagación centrada en jóvenes de movimientos horticultores de la provincia de Buenos 
Aires; esta señala que ser joven es horticultear mientras se estudia y significa tener aún oportunidades de construir un futuro alejado de la quinta; es decir que, aunque las y los jóvenes trabajan allí desde pequeños, la identidad juvenil no se halla adscripta a su condición de productores sino a la condición de estudiantes (Lemmi et al., 2020). Asimismo, una investigación sobre las experiencia formativas de jóvenes que asisten a una escuela rural con albergue en la provincia de Córdoba concluye que la escuela se constituye como el espacio juvenil donde las y los jóvenes pueden expresarse y ser escuchados en sus preocupaciones cotidianas, lo que, a su vez, se traduce en construcciones identitarias renovadoras (Ligorria, 2020). Finalmente, trabajos que abordan experiencias formativas ligadas al Movimiento Campesino de Córdoba permiten advertir diversas situaciones en las escuelas campesinas: al tiempo que es posible hallar experiencias de apropiación por parte de las y los estudiantes de proyectos escolares que articulan prácticas políticas promovidas por el movimiento (Mina \& Flores, 2020), también se advierte que algunos/as estudiantes ponen en cuestión la apropiación de la identidad campesina, en tanto objetivo de la escuela y el movimiento y vinculan lo no urbano con la falta de progreso (Vélez-Funes, 2020).

En la misma línea, ha sido fructífera la recuperación de otros trabajos realizados en América Latina que señalan la importancia que revisten las escuelas como espacios de sociabilidad entre pares en contextos rurales en Perú (Olivera-Rodríguez, 2009) y entre estudiantes de sectores urbano-populares y rurales en México (Ávalos-Romero, 2012; Guerra \& Guerrero, 2004; Hernández, 2007; Weiss, 2015). Frente a los estudios sobre jóvenes que se centran en su condición de alumnos y enfatizan en los procesos de socialización y en la transmisión e interiorización de normas entre generaciones, estos refieren a la importancia de la escuela como «espacio de vida juvenil», enfatizando el encuentro pero también el gusto y el aspecto lúdico de estar junto a pares.

En diálogo con estos aportes, nuestra investigación se sitúa empíricamente en una población rural creada en 1960 como colonia oficial en el Departamento La Paz, ${ }^{2}$ ubicado en el norte de la provincia argentina de Entre Ríos. El norte entrerriano se caracteriza por condiciones agroecológicas y productivas particulares que lo diferencian del resto del territorio de la provincia - mayormente asociado con la llamada región pampeana-, por el alto porcentaje de población rural y la situación socioeconómica y educativa desfavorable de sus habitantes. Los relatos de las y los primeros pobladores de la colonia refieren a una zona «granjera y ganadera», «rodeada de monte nativo» (Escuela Primaria de la colonia, 1983). En relación con las transformaciones vinculadas con la hegemonía

\footnotetext{
${ }^{2}$ De aquí en adelante, nos referiremos a «la colonia» para preservar su nombre.
} 
del modelo de agronegocios, desde mediados de los noventa se destaca el avance de la actividad agrícola ligada a la incorporación de tecnologías de siembra directa y el uso de agroquímicos. Junto a la retracción de la producción ganadera y el avance de la frontera productiva a partir del desmonte, en La Paz se registra la concentración de la propiedad de la tierra y la expulsión de pequeños y medianos productores y pobladores dedicados al trabajo temporario (Dupleich, 2007), lo que, a su vez, aparece en estrecha relación con la profundización de las dificultades de las nuevas generaciones para acceder a la tierra que se remontan a los años de creación de la colonia.

Sin embargo, al tiempo que la cantidad de habitantes disminuye como producto de los cambios estructurales y los crecientes desplazamientos definitivos a la ciudad, entre las transformaciones de las últimas dos décadas en la colonia identificamos la importancia de la creación de la escuela secundaria en el seno de una de sus escuelas primarias. En el marco de la ampliación del tránsito obligatorio por el sistema educativo para las y los jóvenes hasta los 18 años en Argentina y la constitución de la escuela secundaria como el espacio social legítimo para albergar e integrar a este grupo en edad de asistir al nivel (Nobile, 2016), la secundaria de la colonia se constituye como un lugar habitado y en crecimiento; así como también en escenario de disputas sociales e institución fuertemente valorada por las familias al permitir que, a diferencia de la generación que los antecede, las y los egresados de la escuela primaria puedan acceder a la educación secundaria en el lugar de origen (Schmuck, 2019b). Identificamos que la secundaria, además, es el espacio privilegiado de sociabilidad de las y los jóvenes de la zona y entre las actividades que se producen en torno a la institución (como campamentos, torneos deportivos y la noche de egresados) se destaca la Fiesta del Estudiante, que todos los años reúne a jóvenes de las secundarias rurales del norte entrerriano, cuando el 21 de septiembre en Argentina se celebra el día del estudiante y el inicio de la primavera.

En trabajos anteriores desarrollamos como, en el marco de la Fiesta del Estudiante, las y los jóvenes se apropian del discurso hegemónico de la igualdad de oportunidades en la escuela y participan de juegos de inversión de las desigualdades, que significan rupturas respecto de las diferencias económicas y de género que registramos entre estudiantes y sus familias en el espacio social rural (Schmuck, 2019b).

En este artículo nos preguntamos: ¿quiénes son nombrados y se autoadscriben como jóvenes en la colonia?, ¿cómo se configuran sus procesos de identificación - y la construcción de pertenencias y alteridades - en relación con la escuela?, ¿cómo se construye la identificación con el campo y qué nociones sobre el espacio rural atraviesan estos pro- 
cesos? De este modo, nos proponemos analizar los procesos de identificación de las y los jóvenes rurales en vinculación con la educación secundaria.

\section{Método}

Nos posicionamos en la línea de los estudios latinoamericanos de antropología y educación desarrollados desde el enfoque histórico-etnográfico (Achilli, 20oo; Batallán \& Neufeld, 1988; Cragnolino, 2003; Ezpeleta \& Rockwell, 1986), que retoman aportes clásicos de la antropología y la historiografía, rescatando la dimensión cotidiana y experiencial de los sujetos desde una perspectiva relacional: en tensión con las condiciones objetivas del contexto sociohistórico del que los sujetos forman parte, considerando las limitaciones y posibilidades que cada contexto imprime (Cerletti, 2013).

En línea con esta tradición de estudios, entendemos la perspectiva etnográfica en tanto proceso que, a partir de la recuperación de lo cotidiano, permite documentar lo nodocumentado e integrar el conocimiento local (Rockwell, 2009). A lo largo de la investigación, nuestro trabajo de campo ha estado basado fundamentalmente en una búsqueda por acompañar y vivenciar «fragmentos del proceso social en su propio discurrir (...) tejiendo relaciones personales y de confianza» (Quirós, 2014, p. 47), participando de diversas instancias de la vida social de la colonia y, particularmente, de la escuela. Este proceso, que desarrollamos entre 2017 y 2019, implicó la visita periódica a la institución, el trabajo junto con las y los docentes y el personal directivo y, en lo que respecta a los objetivos del presente artículo, con las y los estudiantes. Más que presenciar las clases en espacios áulicos, privilegiamos compartir otros momentos, aprovechando las frecuentes horas libres de estudiantes, las actividades en el predio bajo los árboles que proponían algunas docentes o los momentos en los que las y los jóvenes esperaban su turno para volver a sus casas en los distintos recorridos que hacía el mismo transporte escolar cada mediodía, lo que significaba que permanecieran un tiempo prolongado (a veces horas) en la galería de la escuela.

Aunque en este trabajo también recuperaremos otros fragmentos de nuestra variada participación en la cotidianeidad escolar e incluso conversaciones con jóvenes egresadas de la escuela que ya no viven en la colonia, en lo que refiere a las instancias que han sido fundamentales para comprender las identidades juveniles en las que nos centraremos, interesa destacar que durante dos meses coordinamos en la escuela un taller de comunicación destinado a estudiantes; en este recuperamos nuestro recorrido previo en el cam- 
po de la comunicación social y la experiencia en el dictado de talleres de radio y revista. El taller se integró a las actividades de la escuela de dos formas distintas. En algunos casos, la experiencia se posibilitó a partir de la ausencia de alguna docente y la consiguiente determinación por parte de la directora de que las y los estudiantes debían ocupar el tiempo en la propuesta. En otras oportunidades, invitamos a participar del taller de forma optativa a estudiantes que se encontraban en horas libres, en el momento de espera para ingresar al comedor o luego del mediodía. Esto significó que de los diferentes encuentros participaran alternada - y a veces simultáneamente - un promedio de 10 a 15 jóvenes de los distintos cursos (de $1^{\circ}$ a $6^{\circ}$ año). ${ }^{3}$

En el espacio, que se basó en la metodología de trabajo en talleres de educación y comunicación popular (Schmuck et al., 2021), propusimos rondas de charla y discusión entre jóvenes sobre temáticas de su interés y sobre la vida cotidiana en la escuela y junto a sus familias, al tiempo que los entrenamos en la realización de entrevistas periodísticas y la producción radial. Más allá de los materiales producidos y la formación en sí misma, el acompañamiento en el proceso de producción y las conversaciones que ello habilitó generaron insumos muy provechosos para el análisis: nos permitieron acceder a las rutinas, las actividades educativas y laborales en las que participaban las y los jóvenes y los modos en que construían nociones emergentes sobre la escuela y el espacio rural, que desarrollaremos en el siguiente apartado.

Fue en estos espacios compartidos con jóvenes que fuimos advirtiendo la importancia que le otorgaban a una celebración anual en la que decidimos participar. Así, también formamos parte de las ediciones de 2017 y 2018 de la llamada Fiesta del Estudiante Rural. Durante ambos años estuvimos a cargo del registro audiovisual durante el desarrollo de las jornadas, oficiando - a partir del pedido de la directora - como fotógrafa oficial de la escuela de la colonia y, de este modo, acompañando a las y los estudiantes en los diferentes momentos del desarrollo de las jornadas. Finalmente, en la edición de 2018 estuvimos a cargo junto a una docente (durante tres jornadas escolares que se constituyeron como una continuidad de los talleres de comunicación a los que referimos previamente) de la coordinación de la producción por parte de las y los estudiantes de un videoclip para competir en la Fiesta. Este papel en la Fiesta nos permitió un acceso privilegiado a los modos en que las y los jóvenes querían mostrarse ante las cámaras y, fundamentalmente,

\footnotetext{
${ }^{3}$ La matrícula escolar durante los años de estudio, conformada por jóvenes que viven en un radio promedio de
} 10 kilómetros de la institución, fue de alrededor de 50 estudiantes. 
generó un motivo para acercarnos y compartir el festejo con el estudiantado. ${ }^{4}$ Así, profundizamos la construcción de relaciones de cercanía y confianza con estudiantes, al tiempo que facilitamos espacios de expresión de las y los jóvenes que nos permitieron contar con su propia palabra: creemos que esto ha abonado a nuestro compromiso con la adopción de miradas que escapen a construcciones sociales adultocéntricas a la hora de comprender las juventudes y los sentidos que dan a sus prácticas (Alvarado et al., 2009).

Estas estrategias que fuimos tejiendo fueron resultado de los modos espontáneos de actuar en la cotidianeidad compartida y centralmente de aquello que en la escuela se nos iba proponiendo. De esta manera, la disposición a participar de estos espacios también significó modificar los tiempos y las dinámicas iniciales de la investigación en función de los intereses y deseos de las y los jóvenes y sus docentes, disponiéndonos a tender puentes entre nuestros objetivos y las necesidades advertidas. Así, identificando en cada instancia una posibilidad de trabajo conjunto más que una limitación, asimismo procuramos reconocer a las y los jóvenes como actores sociales competentes, buscando su involucramiento activo y protagónico en los procesos de indagación para cuestionar el tratamiento de los sujetos sociales como objetos (Guerrero \& Milstein, 2021). $\mathrm{Al}$ mismo tiempo, esta particular inmersión en el campo, en la que las diferentes posiciones ocupadas (como tallerista, como fotógrafa) se fueron solapando con nuestro lugar como investigadora, implicó tomar determinados resguardos en virtud de garantizar la ética investigativa: sincerar a la comunidad desde el inicio nuestros propósitos de producción de conocimiento, así como compartir los resultados del trabajo con la institución.

El proceso de análisis, en correspondencia con nuestro enfoque de trabajo, fue cualitativo y etnográfico y no se constituyó como una fase posterior y separada del proceso, sino que estuvo presente desde la formulación de las primeras preguntas y como continua modificación de las preguntas iniciales. Recurrimos a las notas de campo - a veces acompañadas de grabaciones cuando eso era autorizado-, que fuimos ampliando para

\footnotetext{
${ }^{4}$ Cabe señalar que, aunque reconocemos la importancia de los abordajes realizados desde el campo de la antropología visual que hubieran permitido enriquecer la mirada considerando las particularidades del lenguaje audiovisual, en nuestro estudio nos centramos a recuperar el proceso de producción del video y las diferentes actividades de las que participamos en tanto fotógrafa sin avanzar sobre su análisis en tanto productos.

${ }^{5}$ En este sentido, las etnografías colaborativas realizadas con niños, niñas y jóvenes en América Latina, que se desarrollan con un papel protagónico de ellos y ellas en las diferentes instancias del proceso de investigación y buscan posicionar a la escuela como un espacio de producción cultural y de ejercicio político (Guerrero \& Milstein, 2021), podrían constituirse como un camino a seguir en próximos trabajos a partir de la profundización de las estrategias que aquí describimos.
} 
construir registros detallados de la experiencia, realizando recurrentes procesos de lectura, ampliación y ordenamiento considerando el estrecho vínculo entre observación y análisis (Rockwell, 2009). También recuperamos variadas fuentes documentales, entre las que aquí se destaca el archivo escolar, trabajo que consistió en la digitalización y el ordenamiento del archivo de la escuela primaria y la secundaria a partir de documentos producidos por las instituciones y materiales que aportaron las familias. Para el análisis de estas fuentes, tendiendo puentes entre los estudios históricos y antropológicos, nos preguntamos, tanto por su contenido como por los indicios de las prácticas, los procesos de producción del documento, sus marcas de uso y sus silencios (Ginzburg, 1983).

Entendemos que una etnografía también se define por la producción de un tipo de texto: una descripción etnográfica que es producto de un proceso analítico, que «debe conservar e interpretar aquello que se observó, con descripciones analíticas concentradas y detalladas», y presentar conocimiento nuevo y una mayor comprensión de los procesos estudiados que, «si bien dan cuenta de lo que sucedió localmente y de las formas locales de interpretarlo, también deben rendir cuentas a un campo de investigación que se hace preguntas y exige explicaciones, por tentativas que éstas sean» (Rockwell, 2009, p. 184). De este modo, el proceso estuvo atravesado por un juego de relaciones dialécticas entre sistemas conceptuales y la experiencia del trabajo de campo; por lo que las discusiones y categorías teóricas se fueron construyendo, precisando o transformando en su encuentro con conceptos alternativos, sabiendo que «no es posible "olvidar la teoría" para observar, si no se tiene otro lugar desde donde mirar, el conocimiento local» (Rockwell, 2009, p. 94).

En este punto, cabe recuperar algunos hilos conceptuales en torno a los cuales se fue construyendo este permanente proceso de análisis. En primer lugar, hemos buscado apartarnos de las nociones que entienden la juventud en tanto condición natural definida por procesos biológicos o psicobiológicos o como categoría social que se basa exclusivamente en la cuestión etaria. En cambio, nos interesamos por las visiones que consideran la juventud como una construcción social, en tanto condición que no se presenta necesariamente del mismo modo en todas las personas que conforman el grupo poblacional estadísticamente definido como joven, sino que se define en vinculación a contextos históricos y culturales específicos (Chaves, 2010; Pérez-Islas, 2000; Reguillo, 2000). Hablar de «juventudes en plural» implica afirmar que existen diferentes — pero también desiguales - formas de ser joven en función de distintos modos de inserción en la estructura social que es necesario considerar a la luz del lugar de la clase, la residencia, el género, la sexualidad, la etnia, etc., lo que además incide en la construcción de identificaciones 
(Núñez \& Litichever, 2015). Asimismo, recuperamos la mirada que hace hincapié en la concepción de la juventud en tanto construcción social y concepto relacional, pero haciendo especial énfasis en el conflicto entre generaciones (Bourdieu, 1990): las relaciones entre la edad biológica y la edad social son complejas; la juventud (y la vejez) no están naturalmente dadas, sino que se construyen socialmente en la lucha y varían en cada sociedad en diferentes períodos históricos.

Consideramos que el lugar de residencia, estudio o trabajo tampoco basta para definir a las juventudes rurales: lo que es un joven rural o lo que es ser un joven rural se configura según contextos diversos y heterogéneos; no se trata solo de estar en el espacio rural y situarse en un determinado rango de edad o dedicarse a determinada actividad agropecuaria (Kummer \& Colognese, 2013).

En relación con esto último, también consideramos necesario detenernos en las discusiones sobre el concepto de lo rural. En diálogo con las transformaciones en los estudios sociales rurales en la región, en el último cuarto del siglo XX han surgido nuevas concepciones que intentan reconfigurar la mirada hacia el espacio rural cuestionando las definiciones estancas hasta entonces en boga. En relación con estas discusiones, para nuestro trabajo resultó provechosa la reflexión acerca de que, a pesar de haberse estrechado las relaciones y los intercambios entre los espacios urbanos y rurales, en determinados contextos la división sigue existiendo en términos de ingreso, incidencia de pobreza y oportunidades, especialmente en las áreas rurales más dispersas y apartadas de los centros urbanos (Kay, 2009). En ese sentido, en la colonia identificamos continuidades, pero también rupturas respecto a los espacios urbanos, que evidenciamos particularmente en el modo en que las y los pobladores significan el espacio, así como a partir de las construcciones del territorio por parte de las políticas estatales, trabajadores y organizaciones.

\section{Hallazgos}

\section{La Fiesta del Estudiante: las y los jóvenes estudiantes}

A partir de la fundación de la escuela primaria de la colonia en la década de 1960 y desde la creación del secundario a principios de este siglo, para reconstruir el devenir de los proyectos educativos institucionales necesitamos comprender la relación entre distintos procesos, entre los que se encuentran los lineamientos del currículo conformados en los distintos niveles del Estado, las transformaciones en el espacio social rural y las 
diversas concepciones locales sobre la función de la escuela en el campo. En lo que aquí respeta, nos interesa señalar que el proyecto educativo dominante de la secundaria que se expresa en el currículo no se organiza en torno a una modalidad vinculada a lo agropecuario ni a lo técnico-productivo. ${ }^{6}$ Asimismo, en nuestro trabajo concluimos que en relación con las prácticas cotidianas de docentes en su vinculación con los intereses de las diferentes familias, la escuela de la colonia favorece la permanencia hasta finalizar el nivel pero no se propone el arraigo de las jóvenes generaciones al campo.

Entre el estudiantado, aunque no necesariamente haya un reconocimiento del tiempo destinado a estas tareas, algunas jóvenes mujeres participan de actividades relacionadas con la limpieza, el cuidado, las tareas de granja o huerta y el agregado de valor a productos primarios en el ámbito de lo que aparece relacionado con la casa; mientras que, fundamentalmente, algunos varones colaboran o acompañan a sus familias en el trabajo agropecuario asociado al campo, realizando en ambos casos tareas más o menos esporádicas según la demanda de la familia, pero que no interfieren con la asistencia a la escuela. Sin embargo, aunque en determinadas actividades es posible identificar la formación para el trabajo y tareas que guardan algún tipo de vinculación con el medio, desde la institución no se busca articular la producción de saberes con estas experiencias formativas de las y los jóvenes ni incentivar su interés por el trabajo o la vida en el campo. No obstante, como expresa Alicia Civera, es imposible pensar que las escuelas rurales simplemente se posan sobre un espacio diferente al urbano, por lo que interesa indagar acerca de las particularidades de los procesos educativos y escolares en el «mundo rural» (2011, p. 27).

En este sentido, sostenemos que la Fiesta del Estudiante Rural no es cualquier evento de estudiantes en el día de la primavera. Como su nombre lo indica, se trata de un festejo que reúne a distintas escuelas que tienen en común el hecho de emplazarse en contextos rurales y a estudiantes que son referenciados como tales. Además, se trata de una festividad en la que es posible identificar cómo las y los jóvenes se apropian del espacio y los discursos institucionales para producir sus estrategias, propios valores y formas culturales (Levinson, 2002); por lo que consideramos que se trata de un evento propicio para indagar en torno a las particularidades de los procesos de identificación de estas juventudes.

\footnotetext{
${ }^{6}$ Las escuelas primarias de la zona de la colonia y la secundaria no cuentan con una propuesta de formación agropecuaria o agrotécnica. Aunque la secundaria inicialmente funcionó como Bachiller con orientación en Bienes y Servicios Agropecuarios, luego la orientación fue reemplazada por Economía y Administración de Empresas. En este sentido, cabe señalar la diferencia entre las escuelas agrotécnicas, establecimientos de nivel medio cuyo objeto de estudio es «lo agropecuario» y las escuelas rurales —o en contexto rural- son instituciones que inicialmente podemos caracterizar por estar emplazadas en el medio rural (Plencovich, 2014).
} 
Desde 2001, durante la semana del 21 de septiembre ocho escuelas secundarias rurales de los departamentos del norte entrerriano organizan esta jornada deportiva y cultural que se realiza alternadamente en diferentes lugares. Cada año la institución anfitriona define los pormenores de las competencias y elabora un reglamento. Sin embargo, aunque la institución anfitriona asume mayores responsabilidades, todas las escuelas son parte de la organización y disponen desde varias semanas antes de los tiempos y espacios necesarios para organizarse para las diferentes competencias. La jornada propiamente dicha comienza con las actividades deportivas y luego del mediodía incluye actividades artísticas-culturales, competencias de baile, teatro, plástica, video, etc., que varían cada año y finalizan con la habitual elección del rey y la reina de la primavera y un baile.

Aunque el evento parte de una iniciativa de docentes y directivos con acompañamiento ministerial, identificamos que trasciende una propuesta extracurricular y, en las distintas escuelas en que se realiza, cuenta con el apoyo y el protagonismo de las familias de la zona. Las cooperadoras escolares brindan servicio de buffet para juntar dinero para las instituciones y es muy común que las escuelas realicen remodelaciones o arreglos importantes en función del año en el que serán sede del festejo. Durante las jornadas, sobre todo pasado el mediodía, hemos podido advertir cómo familias enteras, que en algunos casos recorren numerosos kilómetros para acompañar a estudiantes de otras escuelas, van llegando con sillones y mates para ser espectadores, pasar la tarde y, en algunos casos, también la noche.

Sin embargo, aunque toda la familia participa, se trata del día en que las y los jóvenes «copamos la escuela» (Registro de campo, escuela de la colonia, agosto de 2017). Motivados por el entusiasmo y las expectativas alrededor del evento, «dejan todo» en la cancha o el escenario durante las jornadas. Podemos ver a jóvenes correr hasta el cansancio durante los partidos, protagonizando expresiones de extrema alegría o tristeza ante cada resultado: abrazos grupales, gritos eufóricos, festejos a los saltos o desconcierto. Aunque en el deporte este despliegue se vuelve más evidente, el resto de las competencias también mantiene al grupo en vilo y el baile se constituye como el cierre más esperado:

En la ronda de preguntas surge el interrogante sobre el momento más divertido del año y al unísono se habla de la Fiesta. Nahuel $l^{7}$ menciona especialmente «el baile». Cuando pregunto qué baile, me explican que es el momento de cierre de la Fiesta y entre risas empiezan a contar anécdotas asociadas a ese momento (...). Tamara cuenta: «Me llevo la ropa en la

\footnotetext{
${ }^{7}$ Los nombres son ficticios para preservar las identidades de las y los jóvenes.
} 
mochi [mochila] porque no me voy a quedar con la remera de la tarde, del vóley y todo eso» (...). Se arma y sale chape [besarse], nos hacemos las lindas [se ríe]. Después sale el tema de la novia de Manuel y entonces me explican que se pusieron de novios en el baile de la primavera del año pasado. «¿Son de salir a otros bailes?», les pregunto. «No tanto, depende. Pero esos son los mejores, con la fiesta de los egresados. Las de acá son lindas; el año pasado vinieron de los otros pueblos», dice Marco. (Registro de campo, talleres de comunicación en la escuela de la colonia, abril de 2017)

Asimismo, el baile nos permite identificar una serie de disputas entre el estudiantado y el mundo adulto. Aunque en las primeras ediciones de la Fiesta se trataba de un momento estipulado dentro del cronograma que proponían las escuelas, con el correr de los años surgieron inconvenientes a partir de la extensión del horario de finalización, con consecuentes problemas con la cobertura del seguro escolar, la logística de traslado del estudiantado en los transportes y quejas de las familias por la tardanza de sus hijos. Por ello, en los últimos años se ha intentado no dilatar el evento luego de la culminación de los certámenes, lo que ha generado descontento entre las y los estudiantes. En 2017, aunque la traffic contratada por la escuela de la colonia había dispuesto un horario de regreso determinado, algunos y algunas jóvenes que se encontraban con sus familias en la localidad vecina - o consiguieron otros medios de transporte, como el vehículo de amigos o una moto- eligieron permanecer en la escuela hasta la finalización del baile, que culminó cerca de la medianoche. En 2018, en cambio, la escuela anfitriona tomó una postura más radical que se tradujo en la inclusión de la siguiente aclaración en el reglamento: «La jornada culmina al finalizar la entrega de premios y recordatorios» (Escuela Secundaria de la colonia, 2018). De este modo, en el cronograma del evento se generó un momento de baile antes de la elección del rey y la reina, incluyendo una clase de baile a cargo de una bailarina arriba del escenario y luego media hora de música acompañada de luces y máquinas de humo que recrearon una pista que se desmontó luego de la finalización (Registro de campo, Fiesta del Estudiante Rural, septiembre de 2018).

Durante los preparativos para la Fiesta en 2017 y 2018 documentamos otras situaciones en las que las y los jóvenes se apropiaban, en forma selectiva, de las propuestas institucionales, entre los que destacamos sus estrategias para destinar más tiempo del premeditado por docentes a las asambleas de estudiantes en las que se conformaban los grupos de trabajo para los eventos, tomando «espacios y tiempos no legítimamente asignados a los estudiantes» (Rockwell, 2011, p. 36). Identificamos, no obstante, el modo en que la participación en la Fiesta también significaba avalar y recuperar algunas reglas del mundo 
adulto, lo que queda claro en las transformaciones que fue sufriendo la propuesta del baile: en esta instancia que implica llevar la escuela como espacio de sociabilidad a su máxima expresión, los procesos de apropiación del estudiantado significan que docentes y directivos busquen nuevamente encuadrar la actividad dentro de parámetros institucionales.

Podemos sostener, entonces, que la Fiesta del Estudiante da cuenta cabalmente del modo en que la condición de juventud se encuentra imbricada con la condición de estudiante y la escuela se constituye como espacio privilegiado de sociabilidad para las y los jóvenes del campo: como lugar en el que disfrutan encontrarse y permanecer, divertirse entre compañeros y amigos en tanto jóvenes. En este punto, nos interesa introducir otro elemento: aunque el acceso y la permanencia en la escuela media en el contexto de estudio se encuentran generalizados, en la colonia también nos encontramos con que son nombrados como jóvenes algunos varones ${ }^{8}$ que han egresado o en algún momento han asistido a la escuela, pero luego han abandonado, principalmente luego de experiencias de repitencia. Se trata de jóvenes que siguen viviendo en el campo y no han «hecho familia», es decir, no tienen pareja con la que viven o hijos y se dedican a trabajar en la zona, generalmente junto a sus padres. En el discurso de docentes y las familias, la diferencia entre incluidos y no incluidos en la educación media se articula con otros discursos y la larga tradición de meritocracia en el sistema educativo de nivel secundario (Chaves et al., 2016). En este sentido, en la colonia se preocupan o lamentan porque estos pocos jóvenes «no han aprovechado la oportunidad», «son haraganes..., con las facilidad que tienen hoy día», «le escapa al libro» (Registros de campo, escuela de la colonia, diciembre de 2018). A pesar de estas manifestaciones - y aunque no es posible afirmar la inexistencia de fronteras sociales en la escuela- para estos jóvenes, aunque no sean estudiantes, el espacio escolar continúa siendo parte de su cotidianeidad.

Identificamos una situación similar en el caso de quienes han egresado y viven en la ciudad cercana u otras localidades donde estudian o trabajan. Considerados jóvenes, visitan periódicamente la casa de sus familias en el campo, generalmente reciben una manutención de padres y madres y no han tenido hijos. Aunque ya no vivan en el espacio rural, sobre todo en los años cercanos a su fecha de egreso, no suelen faltar a la Fiesta (sobre todo a la instancia final del baile) y si, por diversos motivos no pueden estar presentes, están al tanto de los preparativos y contingencias. Así, a pesar de que hayan egresado o

\footnotetext{
8 Por el contrario, aquellas mujeres que se encuentran dentro de un rango etario similar pero se han quedado en la colonia porque se han «juntado» con una pareja e involucrado rápidamente en actividades domésticas o dedicado a la crianza de hijos, no se consideran ni son consideradas jóvenes.
} 
abandonado la institución, estos grupos participan, en tanto jóvenes, de la Fiesta del Estudiante Rural.

\section{Las y los jóvenes del campo}

Como dijimos, entre las competencias centrales durante la Fiesta se encuentran los torneos deportivos para los que se conforman equipos en las escuelas que entrenan todo el año. Esto implica que la escuela de la colonia, que desde mediados de año centra sus esfuerzos en el evento de primavera, también participe de otros torneos enmarcados en diferentes actividades institucionales y deportivas de la zona que muchas veces implican competir con escuelas de la cabecera departamental u otras localidades intermedias. En estas ocasiones, se destaca el orgullo que las y los jóvenes expresan al resaltar los esfuerzos y logros de su escuela y construir una clara rivalidad con los equipos «de la ciudad»:

Vamos a demostrar que venimos del campo, pero eso qué tiene; se la vamos a mandar a guardar (...) arriba los gurises ${ }^{9}$ del campo (...). Nuestra escuela es chica pero poderosa (...); ellos porque se pueden juntar a practicar siempre, tienen la recancha, el reequipo. Acá se nos complica el tema de juntarnos a hacer práctica porque vivimos muy lejos una de otra y no tenemos un lugar cerca de donde vivamos todas las chicas para poder juntarnos. (Registro de campo, talleres de comunicación en la escuela de la colonia, marzo de 2017)

La rivalidad que se construye frente a las escuelas rurales vecinas también reviste suma centralidad durante el desarrollo de las competencias, aunque la identificación entre pares rurales parece erigirse con mayor fuerza a la hora de diferenciarse de las y los estudiantes del campo. En relación con esto, interesa recuperar un registro de una visita que realizamos a una joven egresada de la escuela de la colonia, que se encuentra viviendo en la cabecera departamental:

En la casa de Lucía también estaba una de sus amigas más cercanas, que ha nacido y realizado sus estudios secundarios en [población rural cercana a la colonia], uno de los principales rivales deportivos y en la Fiesta del Estudiante. (...) Más tarde sale el tema del deporte; Lucía dice que cuando eran estudiantes y rivales en las competencias deportivas «nos odiábamos y no nos podíamos ni ver»; aunque cuando luego se reencontraron estudiando Magisterio y enseguida se volvieron amigas porque «las dos somos del campo». (Registro de campo, La Paz, noviembre de 2018)

\footnotetext{
${ }^{9}$ Esta expresión es utilizada en Entre Ríos y algunas otras zonas del nordeste argentino, así como en Uruguay, algunas regiones del sur de Brasil y Paraguay para referirse a niños, niñas o jóvenes.
} 
Aunque en el deporte estos procesos de identificaciones y fronteras adquieren centralidad, también los advertimos en torno al resto de las competencias. Si nos metemos de lleno en la celebración, nos interesa referir al videoclip realizado por la escuela de la colonia en el marco de una de las competencias centrales de la Fiesta del Estudiante en 2018. La producción obtiene el segundo premio en el certamen y genera orgullo y alegría entre estudiantes y docentes. El concurso propone realizar un video que se vincule con el contenido de una canción designada. Participamos de la realización del material desde el primer día en el que, en el marco de una clase de música, un grupo de estudiantes de los últimos años comienza a pensar la historia que se contará en imágenes. Las y los jóvenes, entre intercambios espontáneos poco mediados por las dos adultas que nos encontramos presentes, expresan que muchas veces el miedo que sienten los jóvenes se debe a que se encuentran frente a situaciones desconocidas. Entre distintas propuestas que circulan, las y los jóvenes coinciden en que el video tiene que referir a situaciones que, en palabras de uno de los jóvenes, «tengan que ver nosotros, los estudiantes del campo»:

Durante el intercambio, una estudiante dice que «algunos en [nombre de la ciudad más cercanal piensan que somos brutos porque venimos del campo; campesino bruto piensan». A lo que otro agrega que «se creen que no tenemos celular, que porque somos del campo no podemos tener teléfono» (...). El argumento elegido refiere a la historia de una chica que se muda desde la ciudad a la colonia, comienza a estudiar en la escuela y tiene problemas para adaptarse. Aportan varios y el argumento va tomando forma: luego del conflicto inicial, en el que la chica recién llegada sufre porque no tiene buena señal de internet en el teléfono, no le gusta ensuciarse las manos con tierra haciendo huerta, usa zapatos con taco que se hunden en el barro y le tiene miedo a los caballos que pasan cerca de la institución, el relato termina con un desenlace feliz, en el que la joven logra integrarse al resto del estudiantado al participar de un día de campo y torneos deportivos. (Registro de campo, escuela de la colonia, septiembre de 2018)

El modo deconstrucción de la pertenencia en las y los jóvenes nos remite a pensar, junto con Hall (2003), que las identificaciones pueden comprenderse en relación con un juego de diferencias más que a partir de un conjunto de cualidades predeterminadas que se comparten, implican «un trabajo discursivo, la marcación y ratificación de límites simbólicos, la producción de "efectos de frontera". Necesita lo que queda afuera, su exterior 
constitutivo, para consolidar el proceso» (2003, p. 16)..$^{10}$ Así, la construcción discursiva del nosotros, en tanto jóvenes y estudiantes de la colonia, se constituye a partir de su diferencia con esos otros equipos rivales de la ciudad, estos otros estudiantes de la ciudad.

Por otra parte, de acuerdo con esta historia creada por las y los jóvenes para el videoclip, la chica recién llegada de la ciudad no puede acostumbrarse a lo que emerge como característico de la escuela del campo: la tierra, el barro, los caballos, la falta de señal de internet. Sin embargo, el grupo que realiza este primer guion quiere mostrar que enseguida la joven de ciudad puede sentirse a gusto en el campo, fundamentalmente ante la posibilidad de compartir una tarde al aire libre en un torneo deportivo. Para ello, cuando en otro encuentro el equipo planifica la grabación, se propone que la locación de la última escena sea en «un lugar lindo, que se vea bien el campo» y entonces se piensa en uno de los campos que las familias con mayores recursos suelen prestar para realizar los campamentos o jornadas de fin de año, que disponen de estanques para bañarse, arboledas y predios de césped (Registro de campo, escuela de la colonia, septiembre de 2018).

Como vemos, el repertorio de sentidos que las y los jóvenes estudiantes construyen en torno a la vida y el estudio en el espacio rural, por oposición al urbano, nos remite a la configuración del campo y ciudad como espacios dicotómicos. En estas construcciones encontramos huellas de expresiones que también hallamos entre docentes y directivas, quienes asocian la escuela rural con la tranquilidad, la belleza del lugar y ciertas construcciones idealizadas o bucólicas que nos remiten al discurso escolar rural de principios y mediados del siglo XX (Ascolani, 2000; Gutiérrez, 2002).

En el mismo sentido, nos interesa recuperar uno de los certámenes que integran la propuesta de la Fiesta en 2018, que es la elaboración de un cuadro artístico sobre lienzo. La consigna, según señala el reglamento de la Fiesta, propone tomar una fotografía que luego debe copiarse realizando una representación de un lugar que «represente la identidad cultural o histórica de la zona (...), zonas representativas o icónicas de la escuela o distrito» (Escuela Secundaria de la colonia, 2018):

En el cuadro realizado por la escuela de la colonia se pinta el edificio de la institución rodeado de pastos verdes y árboles. El rasgo característico que me permite notar que se trata de la escuela son los dos característicos pinos al lado del edificio. En primera plana, hay un mate y un libro abierto sobre el pasto. Los cuadros del resto de las escuelas son muy

${ }^{10}$ Hall retoma discusiones de distintos autores. En este punto, resultan significativos los aportes de Butler, Derrida y Laclau en relación con lo que han denominado el exterior/afuera constitutivo. 
parecidos: una huerta escolar rodeada de pastos verdes y una cancha de futbol; árboles, pasto verde y hojas en distintos tonos caídas durante el otoño; un pequeño curso de agua con un árbol en primera plana y de fondo un cerco con plantaciones de color verde; el edificio de la escuela con sus diferentes aberturas y galerías, rodeado de césped verde y árboles, etc. (Registro de campo, Fiesta del Estudiante Rural, septiembre de 2018)

Aquí resulta provechoso recuperar los aportes de Raymond Williams (2011) sobre las ideas e imágenes que a lo largo de la historia se han asociado al campo y la ciudad. El autor señala una operación ligada al proceso de oposición de estos espacios que construye al campo como naturaleza al «suprimir el trabajo campestre y las relaciones de poder a través de las cuales se organiza el trabajo (...) una separación ideológica entre los procesos de explotación rural, que fueron disueltos, en efecto, dentro de un paisaje» (p. 75). Esta constitución del campo como paisaje implica la existencia de un observador que, librado del trabajo, puede permitirse contemplarlo como tal. En el mismo sentido, el mundo rural representado en estas actividades, así como el campo que se construye en el guión del video, se constituyen como un telón de fondo verde que rodea las instituciones. Así como en el guión del video los caballos pasan, totalmente desvinculados del trabajador ganadero a quien acompañan durante sus jornadas, en los cuadros el predio arbolado asume una función de ornamento y disfrute: es un lugar donde tomar mate y leer un libro, un entorno para disfrutar de una tarde de deportes y diversión.

\section{Discusión}

Cuando comenzamos la investigación movilizadas por el interés en las y los jóvenes en los espacios rurales, nuestros interrogantes iniciales no se encontraban ligados al estudio de la escuela secundaria. Sin embargo, desde los inicios del trabajo de campo, la pregunta por las juventudes nos llevó una y otra vez a la escuela secundaria de la colonia, incluso al registrar la preocupación de las familias por aquellos jóvenes que, constituyendo una excepción, no estaban escolarizados. Una vez en la escuela, emergió la fuerte identificación de las y los propios jóvenes con su condición de estudiantes y la importancia de la institución como ámbito de encuentro y diversión, siendo la Fiesta del Estudiante Rural el espacio privilegiado que incluso convoca a quienes no son estudiantes, pero sí se construyen como jóvenes en el campo.

De este modo, los hallazgos del trabajo se corresponden con los diversos estudios sobre juventudes en instituciones educativas que muestran a la escuela como un espacio 
fundamental de encuentro entre pares y sociabilidad juvenil (Chaves, 2010). Particularmente en relación con los estudios sobre juventudes rurales (que, como vimos, a pesar de su impulso en los últimos años se encuentran en relativo estado de vacancia en comparación con el vasto desarrollo de trabajos sobre jóvenes urbanos), la imbricación entre la condición de juventud y la condición de estudiante en la colonia se condice con lo señalado por las investigaciones mencionadas. Situados en variados territorios, estos trabajos que se interesan por diversos jóvenes y estudian desde juventudes de familias campesinas (Barés, 2016) u horticultoras (Lemmi et al., 2020), hasta jóvenes que asisten a escuelas albergues (Ligorria, 2020), del movimiento campesino (Mina \& Flores, 2020; Vélez-Funes, 2020) o se trasladan a instituciones urbanas (Cifuentes-Garzón, 2021), coinciden en señalar la centralidad de escuela secundaria en tanto espacio de vida de las y los jóvenes (Guerra \& Guerrero, 2004; Weiss, 2015) que incide en la configuración - y reconfiguración- de las identidades juveniles.

Asimismo, vimos que las y los jóvenes de la colonia se identifican como estudiantes del campo. En estas construcciones sobre el campo encontramos ecos de otros discursos escolares, que las y los jóvenes transforman en emblema de identificación para construir pertenencias pero también alteridades: esos otros antagónicos, encarnados en las y los jóvenes de la ciudad que fortalecen la identificación con los pares semejantes. En este punto también resuena lo advertido en otros trabajos respecto de los procesos de estigmatización asociados a la pertenencia al campo que, no obstante, implican procesos de resignificación por parte de las y los jóvenes (Barés \& Roa, 2020): lejos de renegar o avergonzarse de su condición de estudiantes del campo y marcando una clara distancia respecto de otros procesos en los que la ruralidad se asocia a lo vergonzante o la falta de progreso (Roa, 2013; Vélez-Funes, 2020), las y los jóvenes de la colonia destacan las bondades del campo y de su escuela que, aunque chica puede ser poderosa; a pesar del barro o de tener problemas con la señal de internet, les permite disfrutar del aire libre y tener acceso a predios de césped, arboledas e incluso alguna jornada a la orilla de un estanque. Así, las y los jóvenes se enorgullecen de la pertenencia que construyen junto a sus pares, disfrutando de la posibilidad de permanecer juntos en la escuela y construyendo apasionamientos en torno a actividades compartidas. La mejor expresión de ello es, nuevamente, la Fiesta del Estudiante Rural, escenario de competencias que son esperadas todo el año y conjugan alegrías y tristezas. A su vez, dado que estudiar las juventudes implica comprenderlas en relación con el contexto social más amplio y en relación -y conflicto- con las otras generaciones, vimos que las tensiones con el mundo adulto durante la Fiesta (particularmente durante el baile), las disputas y las apropiaciones de los tiempos 
institucionales en el marco del festejo también permiten comprender las tramas relacionales en las que se juega la producción de identidades.

En relación con su identificación con el campo, advertimos una particularidad en el modo en que el estudiantado de la colonia construye nociones sobre el espacio rural: las y los jóvenes participan de la configuración de un repertorio de imágenes asociadas al campo como paisaje (Williams, 2009) que se opone a la ciudad. Así, como correlato del proceso de construcción de efectos de frontera (Hall, 2003) respecto a estudiantes de escuelas urbanas que señalamos, el campo aparece como un espacio idílico, asociado a la tranquilidad y la belleza en tanto elementos a contemplar. De esta manera, a diferencia de lo que puede señalarse a propósito de jóvenes que se involucran en la resolución de problemas ambientales (Mina \& Flores, 2020) o defienden el arraigo ligado a las actividades productivas de sus territorios (Cifuentes-Garzón, 2021), en este caso la identificación con el campo no implica una asociación con las actividades o el trabajo rural que realizan muchas de las familias e incluso a veces los propios jóvenes: la identificación no se produce con la figura de agricultor, del ganadero, de la trabajadora de la horticultura o la granja, sino con los elementos de disfrute en el espacio verde como contexto.

Advertimos que, aunque el tránsito por la secundaria incide centralmente en la construcción de la juventud, el egreso no necesariamente se constituye como el paso a la vida adulta; ello nos permite pensar en el carácter de producto sociocultural e histórico de la juventud en tanto categoría social y, al mismo tiempo, en las multiplicidades temporales que marcan las experiencias de las y los sujetos y la inexistencia de etapas sucesivas, ordenadas de modo uniforme en el tiempo. Vimos que la identificación en tanto estudiantes del campo se encontraba presente entre jóvenes egresadas de la escuela secundaria que ya no residen en espacios rurales: sin proyectar necesariamente el retorno o manifestar una relación con determinada actividad productiva rural, estas jóvenes recuerdan con alegría los años de la secundaria y se identifican, en tiempo presente, con el campo entrerriano al señalar que «somos del campo». En este sentido, aunque no entendamos la juventud en términos etarios ni en relación con temporalidades universales, sabemos que algún día las jóvenes dejarán de considerarse y ser consideradas como tales.

En relación con la identificación con el campo, en cambio, surge entonces un nuevo interrogante que anima a continuar los estudios a partir de futuras investigaciones que partan de los aportes aquí desarrollados: ¿es posible pensar en identidades duraderas asociadas al campo en relación con las experiencias de educación secundaria? Señalamos que la existencia de una escuela secundaria en el campo prolonga la estadía, aunque no 
necesariamente se encuentra asociada al arraigo en el espacio rural; no obstante, futuros trabajos centrados en los proyectos de futuro de las y los egresados de la escuela secundaria, considerados en su estrecha relación con las identidades juveniles forjadas en la institución, pero también con las condiciones sociales y familiares de permanencia en el campo, nos permitirán profundizar estas afirmaciones iniciales.

Frente a lo señalado, finalmente, de este trabajo emerge la importancia de la creación y el mantenimiento de instituciones educativas en los espacios rurales que, además de posibilitar el acceso a la educación, vehiculicen la constitución de espacios de encuentro (como la Fiesta del Estudiante Rural) que trasciendan los ámbitos áulicos o las actividades curriculares y se desarrollen en estrecho diálogo con los intereses de las juventudes y sus familias.

\section{Agradecimientos}

Este trabajo no hubiera sido posible sin la disposición y el compromiso de las y los estudiantes de la colonia y sus familias, así como la colaboración de las y los trabajadores de la escuela. La investigación, a su vez, se posibilitó gracias a una beca otorgada por el Consejo Nacional de Investigaciones Científicas y Técnicas de Argentina. Agradecemos, finalmente, a las personas que han evaluado este artículo por sus recomendaciones y aportes al trabajo.

\section{Referencias}

Achilli, E. (200o). Contextos y cotidianeidad escolar fragmentada. Cuadernos de Antropología Social, (12), 11-30.

Alvarado, S., Martínez-Posada, J. \& Muñoz-Gaviria, D. (2009). Contextualización teórica al tema de las juventudes: una mirada desde las ciencias sociales a la juventud. Revista Latinoamericana de Ciencias Sociales, Niñez y Juventud, 7(1), 83-102.

Alvarado, S., Vommaro, P., Patiño, J. A., \& Borelli, S. H. S. (2021). Estudios de juventudes: una revisión de investigaciones en Argentina, Brasil y Colombia, 2011-2019. Revista Latinoamericana de Ciencias Sociales, Niñez y Juventud, 19(2), 1-25. https://doi.org/grdk

Ambrogi, S. (2020). Los relevos generacionales y la formación de jóvenes desde el empresariado agrario pampeano en las últimas décadas. Millcayac, $7(13)$, 389-418.

Ascolani, A (2000). La Pampa pródiga: una imagen del mundo rural para escolares (1920/1945). Mundo Agrario, Revista de Estudios Rurales, 1(1), 1-16. 
Ávalos-Romero, J. (2012). Las conversaciones juveniles en los intersticios del aula. En E. Weiss (Ed.), Jóvenes y bachillerato (pp. 97-124). Asociación Nacional de Universidades e Instituciones de Educación Superior.

Barés, A. (2016). Comunicación, movilidades y espacialidades. Desplazamientos y trayectorias de jóvenes de Norquin Co y Cushamen en la región de la Patagonia argentina. Revista Latinoamericana de Estudios Rurales, 1(1), 105-134.

Barés, A., Hirsch, M., \& Roa, M. L. (2020). Juventudes y ruralidades en Latinoamérica. Hacia un nuevo estado de la cuestión. Millcayac, 7(13), 13-25.

Barés, A. \& Roa, M. L. (2020). Hallarse en el monte y la estepa: corporalidades juveniles rurales. En M. del Mármol, \& M. L. Roa (Comps.), Corporalidades y juventudes: subiendo el volumen (pp. 27-37). Grupo Editor Universitario.

Batallán, G., \& Neufeld, M. R. (1988). Problemas de la antropología y la investigación educacional en América Latina. Cuadernos de Antropología Social, (2),1-6.

Bourdieu, P. (1990). Sociología y cultura. Grijalbo.

Bruniard, R., Jabif, L., \& Bresson, A. (2007). Representaciones y demandas de jóvenes rurales del NOA y NEA argentino sobre sus oportunidades de desarrollo. En R. Bruniard (Coord), La educación de los jóvenes de provincias del NEA y NOA en Argentina (pp. 68-121). Unesco.

Calvo, C., Mariotti, D., \& Ochoa, N. (2015). La situación de la juventud rural en torno al acceso y permanencia en la tierra. Dirección Nacional de Juventud Rural; Secretaría de Coordinación Político Institucional y Emergencia Agropecuaria.

Caputo, L. (2001). Informe de situación. Juventud rural argentina 20oo. Dirección Nacional de la Juventud.

Cerletti, L. (2013). Enfoque etnográfico y formación docente: aportes para el trabajo de enseñanza. Pro-Posições, 24(2), 81-93. https://doi.org/g782

Cifuentes-Garzón, J. E. (2021). Escuela urbana y reconfiguración de identidades en la juventud rural. Revista Colombiana de Educación, 1(82), 131-15o. https://doi.org/gkh28c

Civera, A. (2011). Introducción hacia el estudio de la escuela de los campos latinoamericanos. En A. Civera, G. Alfonseca, \& C. Escalante (Coords.), Campesinos y escolares: la construcción de la escuela en el campo latinoamericano (siglos XIX y XX) (pp. 5-31). El Colegio Mexiquense; Porrúa.

Chaves, M. (2010). Jóvenes, territorios y complicidades: una antropología de la juventud urbana. Espacio Editorial.

Chaves, M., Fuentes, S., \& Vecino, L. (2016). Experiencias juveniles de desigualdad: fronteras y merecimientos en sectores populares, medios altos y altos. Grupo Editor Universitario. 
Cragnolino, E. (2003). Género, trabajo y familia: trayectorias laborales de mujeres de origen campesino en el norte de Córdoba, Argentina. Revista Estudios del Hombre, 16, 212-242.

Cragnolino, E. (2017). Desde las escuelas primarias a las escuelas secundarias campesinas: luchas por la educación pública en Córdoba, Argentina. Educação \& Sociedade, 38(140), 671-688. https://doi.org/10.1590/eso101-73302017177882

Dupleich, J. (2007). El desarrollo territorial: una experiencia en las colonias del norte del departamento La Paz. [Tesis de maestría no publicada]. Universidad Nacional de La Plata.

Escuela Primaria de la colonia. (1983). La historia de la escuela [Material manuscrito producido por docentes y familias de la institución]. Archivo de la escuela primaria de la colonia, La Paz, Entre Ríos, Argentina.

Escuela Secundaria de la colonia. (2018). Reglamento Fiesta del Estudiante Rural [Material producido por docentes institución secundaria rural del Departamento Feliciano]. Archivo de la escuela secundaria de la colonia, La Paz, Entre Ríos, Argentina.

Ezpeleta, J., \& Rockwell, E. (1986). La escuela: relato de un proceso de construcción inconcluso. Revista Colombiana de Educación, (12). https://doi.org/g784

Gili-Diez, V. (2019). Territorialidad chacarera en disputa: reflexiones acerca de jóvenes chacareros de médano de oro. Tramas Sociales, 1(1), 24-41.

Ginzburg, C. (1983). Señales: raíces de un paradigma indiciario. En A. Gargani (Comp.), Crisis de la razón. Siglo XXI.

Gómez, C. \& Porta, M. P. (2018). Educación rural y empleo: expectativas e itinerarios de inserción laboral en egresados de las Escuelas de la Familia Agrícola (EFAs) de Chaco y Corrientes. En Actas VI Renija GT12 (pp. 2-15). Red de Investigadores en Juventudes de Argentina.

González-Cangas, Y. (2003). Juventud rural: trayectorias teóricas y dilemas identitarios. Nueva Antropología, $19(63), 153^{-175}$.

Guerra, I., \& Guerrero, E. (2004). ¿Qué sentido tiene el bachillerato?: una visión desde los jóvenes. Universidad Pedagógica Nacional.

Guerrero, A. L., \& Milstein, D. J. (2021). Lecturas de etnografías colaborativas con niñas, niños y jóvenes en contextos educativos latinoamericanos. Magis, 14, 1-33. https:// doi.org/10.11144/Javeriana.m14.lecn

Gutiérrez, V. T. (2002). El mundo rural pampeano en el discurso educativo peronista. Buenos Aires, 1946-1955. Cuadernos del Piea, 16, 5-34.

Hall, S. (2003). Introducción. «¿Quién necesita 〈identidad»?». En S. Hall, \& P. Du Gay (Ed.), Cuestiones de identidad cultural (pp. 13-39). Amorrortu. 
Hernández, J. (2007). La formación de la identidad en el bachillerato: reflexividad y marcos morales [Tesis de doctorado]. Centro de Investigación y de Estudios Avanzados del Instituto Politécnico Nacional; DIE; Cinvestav.

Hirsch, M. (2020). Jóvenes y proyectos de futuro: entre la educación superior y el trabajo en Cañuelas, provincia de Buenos Aires. Estudios Rurales, 10(19), 1-16.

Kay, C. (2009). Estudios rurales en América Latina en el periodo de globalización neoliberal: ¿una nueva ruralidad? Revista Mexicana de Sociología, 71(4), 607-645.

Kessler, G. (2007). Juventud rural en América latina: panorama de las investigaciones actuales en educación, desarrollo rural y juventud. En R. Bruniard (Coord.), La educación de los jóvenes de provincias del NEA y NOA en Argentina (pp. 16-67). Unesco.

Kummer, R., \& Colognese, S. (2013). Juventude rural no Brasil: entre ficar e partir. Tempo da Ciência, 20(39), 201-220.

Lemmi, S., Morzilli, M., \& Castro, A. (2020). Jóvenes que horticultean, adultos/as horticultores/as. Aproximaciones al sentido de juventud en familias migrantes bolivianas que se dedican a la horticultura en el Gran La Plata. Millcayac, $7(13), 141-172$.

Levinson, B. (2002). Todos somos iguales: cultura y aspiración estudiantil en una escuela secundaria mexicana. Santillana.

Ligorria, V. (2020). Alejarse y extrañar para poder estudiar: trayectorias de jóvenes en una escuela secundaria rural con albergue mixto. Millcayac, 7(13), 323-344.

Mina, M. R., \& Flores, H. A. (2020). El problema del agua: un abordaje etnográfico en torno a los proyectos de futuro de los jóvenes en el Movimiento Campesino de Córdoba. Millcayac, $7(13), 305-322$.

Nobile, M. (2016). La escuela secundaria obligatoria en Argentina: desafíos pendientes para la integración de todos los jóvenes. Última Década, 24(44), 109-131. https:// doi.org/10.4067/So718-22362016000100005

Núñez, P., \& Litichever, L. (2015). Radiografías de la experiencia escolar. ser joven(es) en la escuela. Grupo Editor Universitario. https://doi.org/10.2307/j.ctvtxw2d6

Olivera-Rodríguez, I. (2009). Los sentidos de la escolaridad: o la relación juventud ruralescuela frente a los procesos de exclusión. Revista Peruana de Investigación Educativa, 1(1), 61-9o. https://doi.org/10.34236/rpie.vii1.3

Padawer, A., Rodríguez-Celín, L., Canciani, M. L, Greco, J., \& Soto, A. (2015). La obligatoriedad de la secundaria: las modalidades de educación intercultural bilingüe y educación rural en el sudoeste misionero. Boletín de Antropología y Educación, 6(9), 71-78.

Pérez-Islas, J. A. (2000). Jóvenes e instituciones en México, 1994-200o: actores, políticas y programas. SEP; Instituto Mexicano de la Juventud. 
Plencovich, M. C. (2014). Sistema educativo y educación agraria: deriva e inclusión. Fundación Ciccus.

Quirós, J. (2014). Etnografiar mundos vívidos. desafíos de trabajo de campo, escritura y enseñanza en antropología. Publicar, 12(17), 47-65.

Reguillo, R. (2000). Emergencia de culturas juveniles: estrategias del desencanto. Norma.

Roa, M. L. (2013). Sufriendo en el yerbal... Los procesos de self en jóvenes de familias tareferas. Revista Latinoamericana de Ciencias Sociales, Niñez y Juventud, 11(1), 171-184.

Rockwell, E. (2009). La experiencia etnográfica: historia y cultura en los procesos educativos. Paidós.

Rockwell, E. (2011). Los niños en los intersticios de la cotidianeidad escolar: ¿resistencia, apropiación o subversión? En G. Batallán, \& M. R. Neufeld (Coords.), Discusiones sobre infancia y adolescencia: niños y jóvenes, dentro y fuera de la escuela (pp. 27-52). Biblos.

Román, M. (2003). Los jóvenes rurales en Argentina: elementos para una estrategia de desarrollo rural. Ministerio de la Producción; Secretaría de Agricultura, Ganadería, Pesca y Alimentos; Dirección de Desarrollo Agropecuario; Proinder.

Román, M. (2011). Juventud en áreas rurales de Argentina: impacto de los cambios ocurridos en la década del noventa [Tesis de doctorado]. Universidad de Buenos Aires.

Romero-Acuña, M. (2018). Ciudad, ruralidad isleña y políticas públicas: experiencias formativas de los jóvenes en el Delta del Paraná (Rosario-Argentina). Kult-Ur, 5(10), 170-189. https://doi.org/10.6035/kult-ur.2018.5.10.7

Schmuck, M. E. (2019a). Juventudes en plural, territorios en transformación: hacia un estado del arte de los estudios sobre juventudes rurales en Argentina. PÓS, 14(1), 38-56.

Schmuck, M. E., (2019b). Jóvenes rurales en la escuela secundaria del campo: una etnografía sobre estudiantes en el norte entrerriano. Irice, 35(1), 29-158.

Schmuck, M. E. (2020). Entre géneros y generaciones: transformaciones en las experiencias formativas de familias del espacio rural del norte entrerriano. Tiempo de Gestión, 16(29), 67-97.

Schmuck, M. E., Marioni, L., \& Giangelici, J. (2021). «¿Se puede ser un medio y un taller?»: tensiones entre comunicación y educación popular en El Surco del Oeste. Educación $y$ Vínculos, (7), 197-216.

Vélez-Funes, C. (2020). Jóvenes rurales del noroeste cordobés. Límites y posibilidades para permanecer en el campo. Millcayac, 7(13), 173-196.

Weiss, E. (2015). Más allá de la socialización y de la sociabilidad: jóvenes y bachillerato en México. Educação e Pesquisa, 41, 1257-1272. https://doi.org/g785

Williams, R. (2009). Marxismo y literatura. Las Cuarenta.

Williams, R. (2011). El campo y la ciudad. Paidós. 